\title{
Extraction of guaiacol from model pyrolytic sugar stream with ionic liquids
}

\author{
Xiaohua Li, Sascha R. A. Kersten and Boelo Schuur* \\ University of Twente, Sustainable Process Technology Group, Faculty of Science and \\ Technology, Postbus 217, 7500AE Enschede, The Netherlands.
}

*Corresponding author: Email: b.schuur@utwente.nl, Tel: +31 534892891.

\section{$\sigma$-Profiles of guaiacol, levoglucosan, water and IL anions obtained by COSMO-RS}

$\sigma$-Profiles $p(\sigma)$ describe the quantity of surface with screening charge polarity $\sigma$ on the surface of the molecule. The $\sigma$-profiles and COSMO-cavities of guaiacol, levoglucosan and water are shown in Figure S1. The entire $\sigma$-profile of water spans the range of $-0.020<\mathrm{e} / \AA^{2}<$ 0.022. Two major peaks are arising from the negative charged regions of the oxygen atom (thus at a positive value of $\sigma$ due to the larger electron density) and from the positively charged hydrogen atoms (thus at negative values of $\sigma$ ). Generally, the $\sigma$-regions beyond \pm 0.01 $\mathrm{e} / \AA^{2}$ are considered as strongly polar and potentially hydrogen bonding (HB), and the rest as weakly polar or non-polar. Therefore, the surface with charge below $-0.01 \mathrm{e} / \AA^{2}$ is considered as a $\mathrm{HB}$ donor region, while the HB acceptor region is located above $+0.01 \mathrm{e} / \AA^{2}$. The $\sigma$ profile of guaiacol shows its hydrophobicity, since the $\sigma$-surface is mainly located in the nonpolar region. However, the hydroxyl hydrogen shows a peak at $-0.017 \mathrm{e} / \AA^{2}$, which can be HB donor. As for levoglucosan, the peak at $+0.014 \mathrm{e} / \AA^{2}$ results from the negative charged surfaces of the hydroxyl oxygens, and the peak at $-0.015 \mathrm{e} / \AA^{2}$ is caused by the electron deficiency on the surfaces of hydroxyl hydrogen atoms. Having three HB donor and three HB 
acceptor regions, and in addition a smaller surface area in the hydrophobic region than guaiacol, it follows that levoglucosan should be much more hydrophilic than guaiacol.

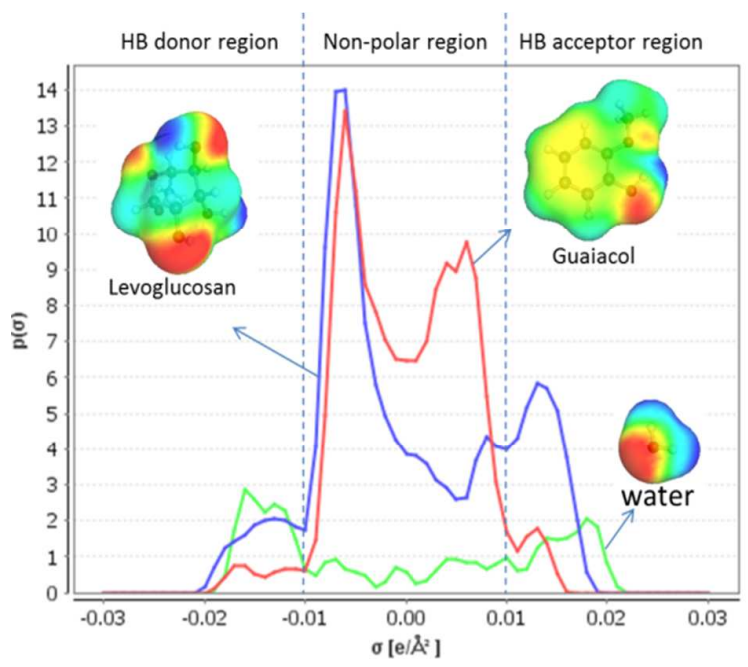

Figure S1. $\sigma$-Profiles and COSMO-cavities of guaiacol, levoglucosan and water. The blue color indicates a negative screening charge resulting from positive partial charges within the molecule. The red color indicates a positive screening charge resulting from electronegative surface elements, and green corresponds to almost neutral charges

$p(\sigma)$ of IL anions obtained by COSMO-RS are shown in Figure S2. From the $\sigma$-profiles, it follows that the polarity of the anions ranks as follows: Phos $>\mathrm{Cl}>\mathrm{N}(\mathrm{CN})_{2}>\mathrm{B}(\mathrm{CN})_{4}>$ $\mathrm{NTf}_{2}>\mathrm{PF}_{6}$

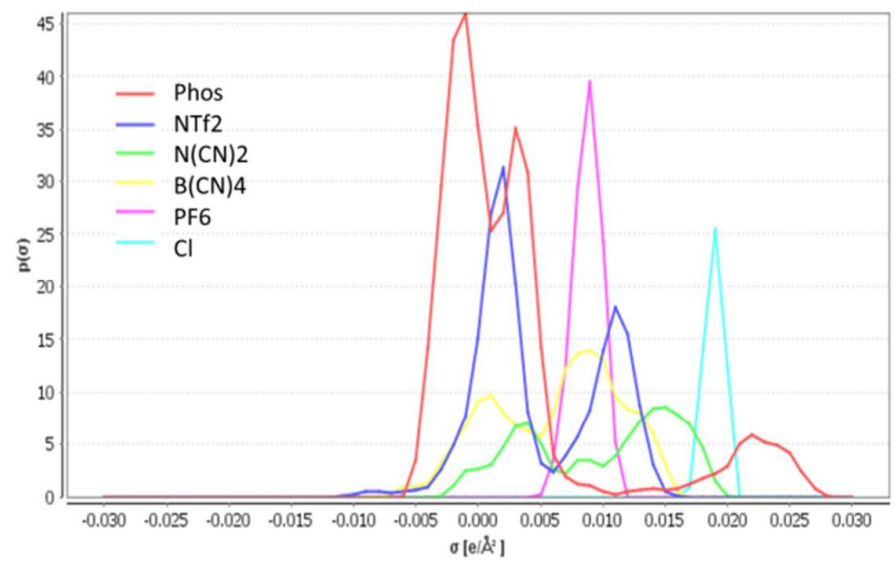

Figure S2. $\sigma$-Profiles of IL anions obtained by COSMO-RS 


\section{Liquid-liquid equilibrium data for ternary system EA + guaiacol + H2O}

The experimental ternary phase diagram was developed by contacting water with guaiacol solutions in ethyl acetate (EA) with guaiacol mass fractions of $0.05<\mathrm{x}<0.95$ at room temperature at a mass ratio of 0.5 (EA phase / water). After mixing for 30 minutes, the phases were separated by centrifuging for $5 \mathrm{~min}$ at $9000 \mathrm{rpm}$. Both phases were analyzed by HPLC to obtain the guaiacol concentration, and by Karl Fischer to get the $\mathrm{H}_{2} \mathrm{O}$ concentration. The ternary equilibrium data are listed in Table S1.

Table S1. Experimental data (relative standard deviation $<3 \%$ ) for ternary system EA +

$$
\text { guaiacol }+\mathrm{H}_{2} \mathrm{O} \text { based on mole fraction }
$$

\begin{tabular}{cccccc}
\hline$x_{E A}^{a q}$ & $x_{\text {guai }}^{a q}$ & $x_{H_{2} O}^{a q}$ & $x_{E A}^{E A}$ & $x_{\text {guai }}^{E A}$ & $x_{H_{2} O}^{E A}$ \\
\hline 0.0011 & 0.0031 & 0.9958 & 0.0506 & 0.6962 & 0.2532 \\
0.0024 & 0.0028 & 0.9948 & 0.1307 & 0.6265 & 0.2428 \\
0.0038 & 0.0026 & 0.9936 & 0.203 & 0.5587 & 0.2383 \\
0.0055 & 0.0023 & 0.9923 & 0.2791 & 0.4771 & 0.2438 \\
0.0072 & 0.0019 & 0.9909 & 0.363 & 0.4067 & 0.2303 \\
0.0091 & 0.0016 & 0.9894 & 0.4494 & 0.3275 & 0.2231 \\
0.0108 & 0.0012 & 0.9880 & 0.5370 & 0.2510 & 0.2120 \\
0.0127 & 0.0008 & 0.9865 & 0.6305 & 0.1720 & 0.1974 \\
0.0144 & 0.0004 & 0.9852 & 0.7316 & 0.0948 & 0.1736 \\
0.0148 & 0.0003 & 0.9849 & 0.7851 & 0.0606 & 0.1544
\end{tabular}

\section{NRTL model fitting for EA + guaiacol $+\mathrm{H}_{2} \mathrm{O}$}


The full ternary phase diagram was experimentally investigated, and a thermodynamic description of the system $\mathrm{EA}+$ guaiacol $+\mathrm{H}_{2} \mathrm{O}$ was developed using the NRTL model.

This included fitting the binary interaction parameters in equation (S1) for the NRTL model, which was done using the ASPEN PLUS Data Regression tool. The regressed parameters are presented in Table S2.

$\tau_{i j}=a_{i j}+\frac{b_{i j}}{T}, G_{i j}=\exp \left(\alpha_{i j} \tau_{i j}\right), \alpha_{i j}=\alpha_{j i}$.

Table S2. NRTL binary parameters for the ternary LLE data of the system (EA + guaiacol $+\mathrm{H} 2 \mathrm{O})$

\begin{tabular}{cccccc}
\hline System & $\mathrm{a}_{12}$ & $\mathrm{a}_{21}$ & $\mathrm{~b}_{12}$ & $\mathrm{~b}_{21}$ & $\alpha$ \\
\hline EA (1) - H2O (2) ${ }^{\mathrm{a}}$ & $-3,7198$ & 9,4632 & 1286,14 & $-1705,6$ & 0,2 \\
EA (1) - Guaiacol (2) & 0 & 0 & 1487,5 & 265,7 & 0,3 \\
Guaiacol (1) -H2O (2) & 0 & 0 & $-1.859,7$ & 1390,6 & $-0,055$
\end{tabular}

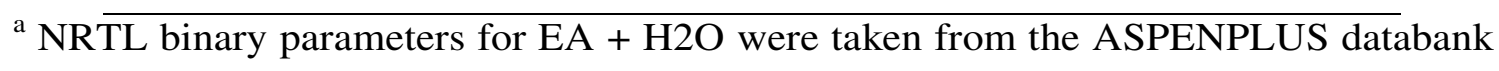
“APV84 LLE-ASPEN” in version 8.4.

The rRMSD was calculated to describe the goodness of the NRTL model agreement with the experimental data. The $r R M S D$ is defined as

$$
r R M S D=\sqrt{\frac{\sum_{i}^{N}\left(\frac{x^{e x p}-x c a l}{x^{e x p}}\right)^{2}}{n}}
$$

where $n$ represents the number of data points and $x$ is the mole fraction. From the agreement between the experimental and calculated ternary diagrams in Figure 5, it was concluded that the NRTL model properly describes the experimental ternary data of system EA + guaiacol + $\mathrm{H}_{2} \mathrm{O}$, this was confirmed by the $r R M S D$ of 0.041 . 


\section{Minimum S/F ratio calculation for Process with EA solvent}

All the calculations are based on the following four assumptions.

1) the resulting extract flow approximately equals the solvent flow and the raffinate flow approximately equals the feed flow;

2) the incoming feed is in equilibrium with the outgoing extract stream;

3) $99.995 \%$ of the solute was extracted by the solvent;

4) the extraction was operated at $20{ }^{\circ} \mathrm{C}$

According to the equation S3

$S_{\text {min }}=F \frac{X_{\text {in }}-X_{\text {out }}}{Y_{\text {out }, \text { max }}-Y_{\text {in }}} \quad$ with $Y_{\text {out }, \text { max }}=D X_{\text {in }}$

Where $S_{\text {min }}$ is the minimum solvent, $F$ is Feed, $X_{\text {in }}, X_{\text {out }}, Y_{\text {out,max }}, Y_{\text {in }}$ is solute weigh ratio in feed, raffinate, extract, solvent respectively, and D is the distribution coefficient.

In our case, $X_{\text {in }}$ is 0.015 and D is 44.7 , then $Y_{\text {out,max }}$ can be calculated and therefore minimum solvent $\mathrm{S} / \mathrm{F}$ ratio is 0,022 . A reasonable starting value for $\mathrm{S} / \mathrm{F}$ is usually $1.5 * \mathrm{~S} / \mathrm{Fmin}$ which is 0.034 . However, the solubility of EA in water is $83 \mathrm{~g} / \mathrm{L}$, so to get good phase separation after extraction, $\mathrm{S} / \mathrm{F}$ ratio 0.2 was used to calculate other streams.

\section{Optimization for DST1 and DST2 columns in Process with EA solvent}

To calculate the energy consumption for ethyl acetate recovery by distillation, the distillation columns were simulated in ASPEN Plus. Without thoroughly optimizing the required number of equilibrium stages (NTS), the DST1 column was simulated with NTS $=20$, and the feed stream added above the $10^{\text {th }}$ stage. The liquid compositions of the three components through the column are shown in Figure S3. From the graph, it can be seen that stages 11-20 do not contribute to the separation. Therefore the NTS was decreased 
and after some iterations, NTS was set to 8 , as there were useless stages when NTS $>8$. The feed stream was added above the $5^{\text {th }}$ stage. The liquid compositions of three components throughout the column are shown in Figure S4 for NTS $=8$.

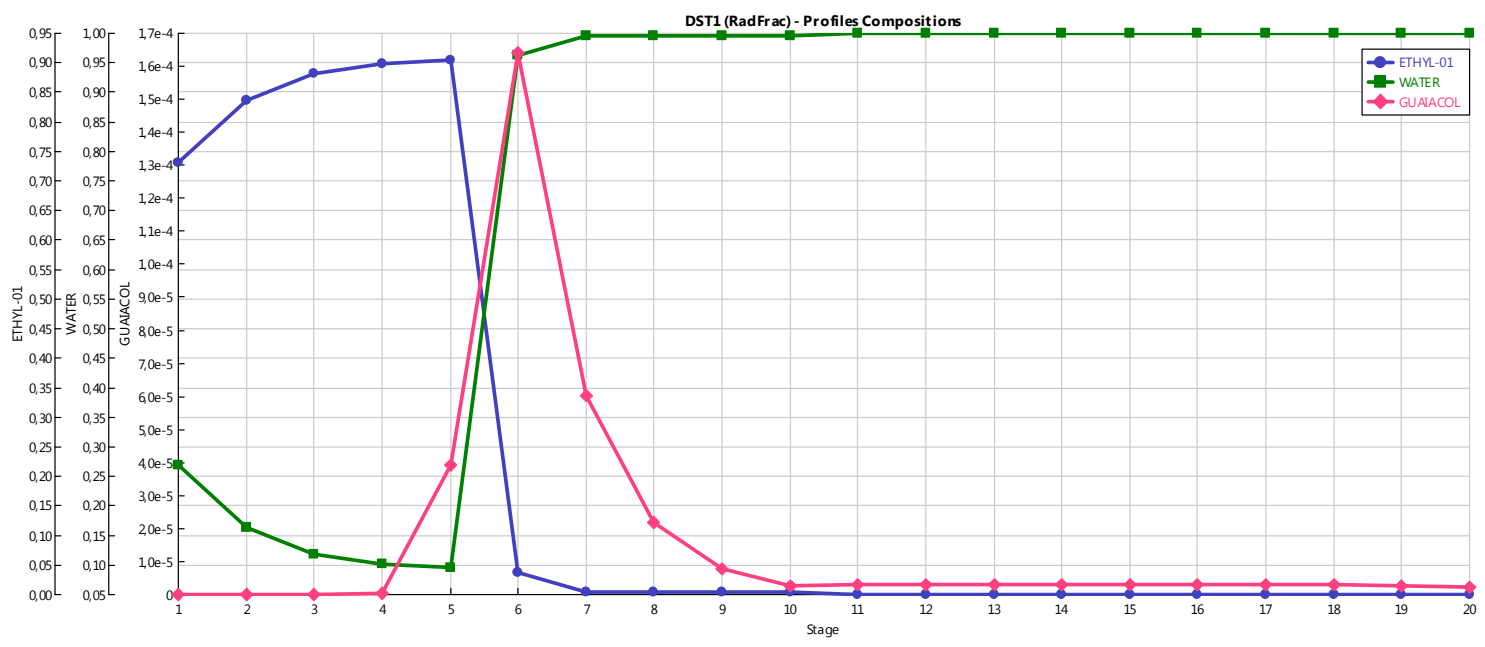

Figure S3. The liquid compositions of the three components throughout the DST1 column for NTS $=20$ (stage 1 is the top stage $)$

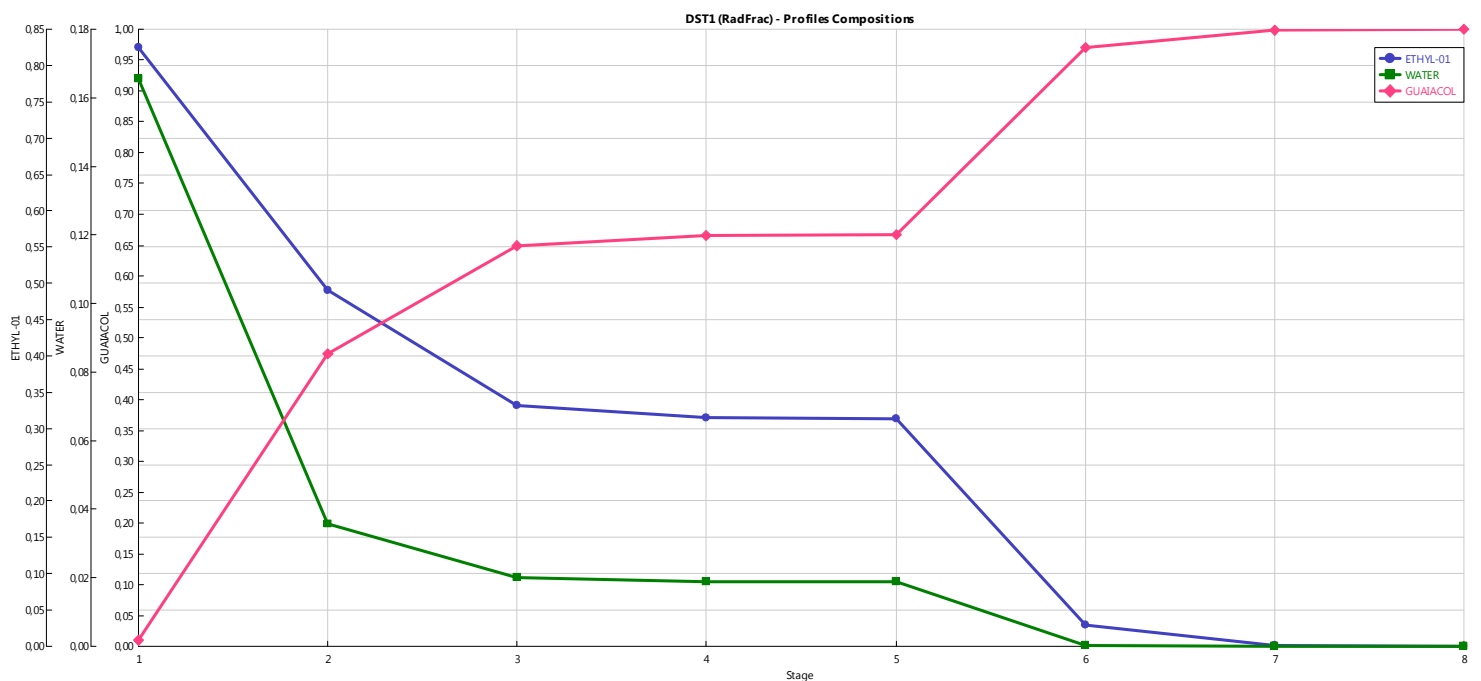

Figure S4. The liquid compositions of the three components throughout the DST1 column for NTS $=8$ (stage 1 is the top stage)

The distillation column DST1 was modeled for different distillate rates and reflux ratios (R) to investigate whether these ratios have a large effect on the solvent recovery 
efficiency and reboiler heat duty, keeping the makeup solvent flow EA $=1 \mathrm{~kg} / \mathrm{h}$, feed flow $=1000 \mathrm{~kg} / \mathrm{h}$ and for DST2 column distillate rate $=86 \mathrm{~kg} / \mathrm{h}$ and $\mathrm{R}=0.5$. Firstly, the $\mathrm{R}$ of DST1 was set as 0.1 to evaluate the effect of distillate rate. The results for solvent (EA) recovery efficiency and reboiler heat duty for DST1 column as function of distillate rate are shown in Figure S5. Both the solvent recovery efficiency and reboiler heat duty increase with the increase of distillate rate. To recovery $99 \%$ of solvent EA, the distillate rate needs to be $124 \mathrm{~kg} / \mathrm{h}$.
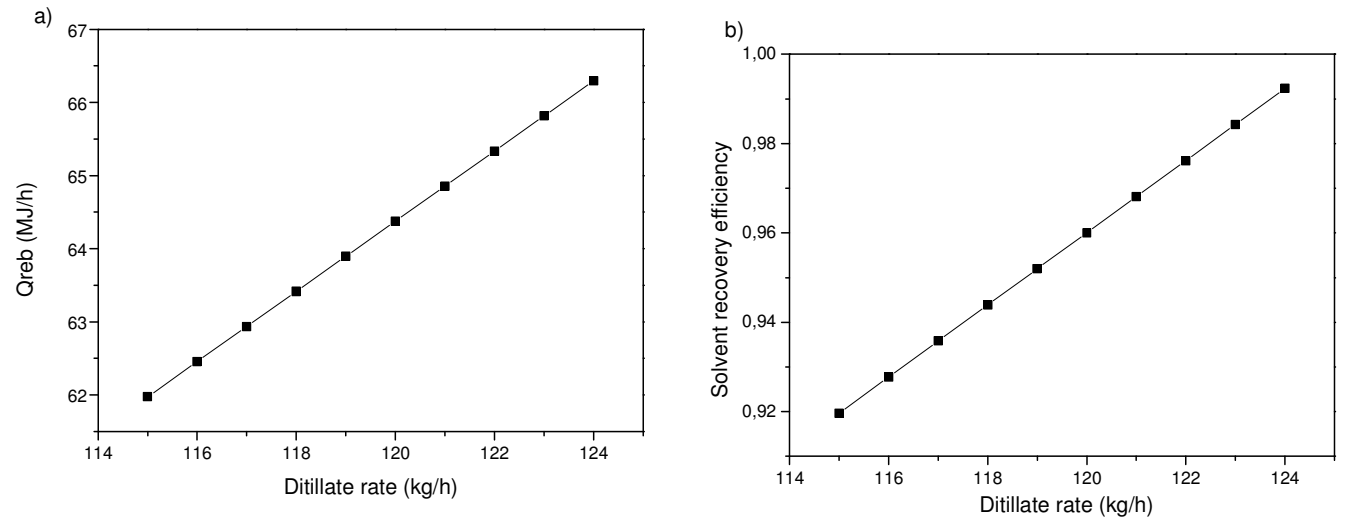

Figure S5. a) reboiler heat duty Qreb and b) solvent recovery efficiency of DST1 column as function of Distillate rate, based on $\mathrm{R}=0.1$ for DST1.

Secondly, the effect of $\mathrm{R}$ was evaluated based on the distillate rate $124 \mathrm{~kg} / \mathrm{h}$. The results for solvent recovery efficiency and reboiler heat duty for DST1 column as function of R are shown in Figure S6. The solvent recovery efficiency was hardly dependent on the R and kept at $99.23 \%$, while the Qreb increased with increasing R. Therefore, the minimum R 0.1 was chosen for DST1. 

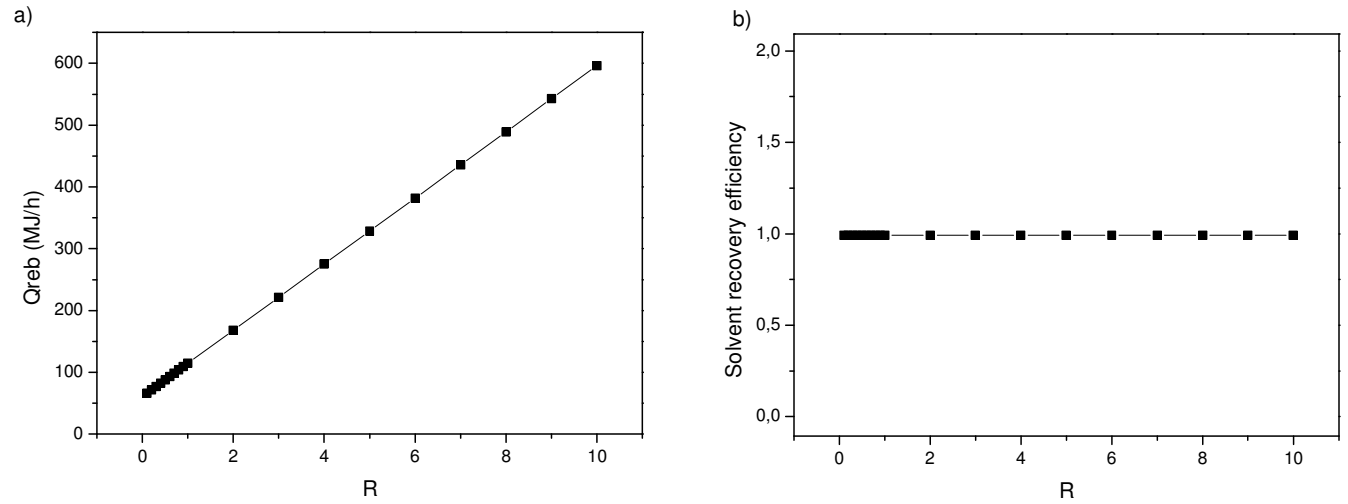

Figure S6. a) reboiler heat duty Qreb and b) solvent recovery efficiency of DST1 column as function of reflux ratio $\mathrm{R}$, based on distillate rate $124 \mathrm{~kg} / \mathrm{h}$ for DST1.

The optimization of DST2 column was done by the same method as for DST1 column, the NTS 8 was used and the feed stream was added on the stage 5 . Then the same method was used to optimize the R for DST2 column, based on a distillate rate of $86 \mathrm{~kg} / \mathrm{h}$ which is shown in Figure S7. R 0.5 was needed to recovery $99.9 \%$ solvent EA. When $\mathrm{R}$ was set as 0.5 , the distillate rate could only be 87 or $86 \mathrm{~kg} / \mathrm{h}$ to make the process continuously running. A distillate rate of $86 \mathrm{~kg} / \mathrm{h}$ was chosen for DST2, since it meets the requirement of $99.9 \%$ solvent recovery and shows a lower Qreb.
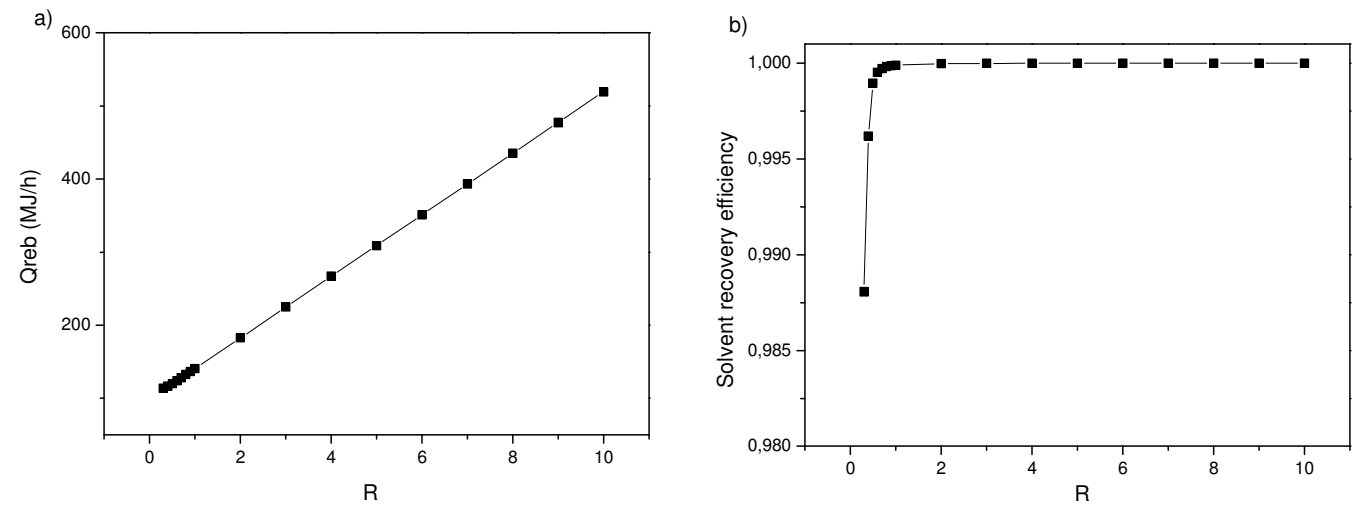

Figure S7. a) reboiler heat duty Qreb and b) solvent recovery efficiency of DST2 column as function of reflux ratio $\mathrm{R}$, based on distillate rate $86 \mathrm{~kg} / \mathrm{h}$ for DST2. 


\section{Liquid-liquid equilibrium data for ternary system $\mathbf{P}_{666,14}\left[\mathbf{N}(\mathrm{CN})_{2}\right]+$ guaiacol + $\mathbf{H}_{2} \mathbf{O}$}

Liquid-liquid extraction experiments for this system were similar as for system EA + guaiacol $+\mathrm{H}_{2} \mathrm{O} . \mathrm{P}_{666,14}\left[\mathrm{~N}(\mathrm{CN})_{2}\right]$ and guaiacol solutions in the IL with guaiacol mass fractions of $0.05<\mathrm{x}<0.95$ were mixed with water at a mass ratio of 0.5 (IL phase / water) for $30 \mathrm{~min}$, then centrifuged for $5 \mathrm{~min}$ to get two separated phases. The aqueous phase was analyzed by HPLC to determine the guaiacol content, and both phased were analyzed by Karl Fischer to determine the water content. The guaiacol content in the IL phase was determined by mass balance. The equilibrium results are shown in Table S3.

Table S3. Experimental data for ternary system $\mathrm{P}_{666,14}\left[\mathrm{~N}(\mathrm{CN})_{2}\right]+$ guaiacol $+\mathrm{H} 2 \mathrm{O}$ based on mass fraction (relative standard deviations are lower than $3 \%$ )

\begin{tabular}{|c|c|c|c|c|c|c|}
\hline $\begin{array}{l}x_{I L}^{a q} \\
(\mathrm{ppm})\end{array}$ & $x_{g u a i}^{a q}$ & $x_{\mathrm{H}_{2} \mathrm{O}}^{a q}$ & $x_{I L}^{I L}$ & $x_{\text {guai }}^{I L}$ & $x_{\mathrm{H}_{2} \mathrm{O}}^{I L}$ & $D_{\text {guai }}$ \\
\hline $46 \pm 10$ & 0.00035 & 0.9996 & 0.9237 & 0.0496 & 0.0267 & 142 \\
\hline $50 \pm 2$ & 0.00087 & 0.9991 & 0.8785 & 0.0977 & 0.0238 & 112 \\
\hline $53 \pm 0$ & 0.00244 & 0.9975 & 0.7912 & 0.1905 & 0.0183 & 78 \\
\hline $50 \pm 2$ & 0.00566 & 0.9943 & 0.6907 & 0.2928 & 0.0165 & 52 \\
\hline $62 \pm 5$ & 0.01033 & 0.9896 & 0.5884 & 0.3961 & 0.0155 & 38 \\
\hline $83 \pm 1$ & 0.0149 & 0.9850 & 0.4842 & 0.4978 & 0.0181 & 33 \\
\hline $83 \pm 4$ & 0.01853 & 0.9814 & 0.3895 & 0.5901 & 0.0205 & 32 \\
\hline $80 \pm 1$ & 0.02113 & 0.9788 & 0.2719 & 0.7053 & 0.0228 & 33 \\
\hline
\end{tabular}




$\begin{array}{lllllll}80 \pm 2 & 0.02272 & 0.9772 & 0.1433 & 0.8277 & 0.0290 & 36 \\ 89 \pm 1 & 0.02421 & 0.9757 & 0.0989 & 0.8655 & 0.0355 & 36\end{array}$

\section{NTS of extraction column for process with IL as solvent}

The NTS of an extraction process with IL as solvent was calculated using equations (S4) and (S5)

$$
\begin{aligned}
& \frac{x_{R}}{x_{F}}=\frac{E-1}{E^{N+1}-1} \\
& E=D \frac{S}{F}
\end{aligned}
$$

Where $x_{\mathrm{R}}$ and $x_{\mathrm{F}}$ are the guaiacol concentrations in the raffinate and feed stream, respectively, and $\mathrm{N}$ is the number of stages. $\mathrm{E}$ is the extraction factor and $\mathrm{S} / \mathrm{F}$ is solvent to feed ratio. $\mathrm{D}$ is the distribution coefficient, for which 50 was taken as it is the average value for a multistage extraction.

In the extraction case, $x_{\mathrm{F}}$ was 0.015 , the required $x_{\mathrm{R}}$ was 0.00005 and $\mathrm{S} / \mathrm{F}$ was 0.045 , then the calculated $\mathrm{N}$ amounts to 6.31 . Therefore, the required NTS was 7.

\section{Thermodynamics properties of water, guaiacol and $\mathbf{P}_{666,14}\left[\mathrm{~N}(\mathrm{CN})_{2}\right]$}

Thermodynamics properties of water, guaiacol and $\mathrm{P}_{666,14}\left[\mathrm{~N}(\mathrm{CN})_{2}\right]$ are shown in Table S4.

Table S4. Thermodynamics properties of water, guaiacol and $\mathrm{P}_{666,14}\left[\mathrm{~N}(\mathrm{CN})_{2}\right]$ 


\begin{tabular}{cccc}
\hline Compounds & $\begin{array}{c}\mathrm{C}_{\mathrm{p}} \\
(\mathrm{J} /(\mathrm{g} \cdot \mathrm{K}))\end{array}$ & $\begin{array}{c}\Delta \mathrm{H}_{\mathrm{vap}} \\
(\mathrm{J} / \mathrm{g})\end{array}$ & $\mathrm{T}_{b}\left({ }^{\circ} \mathrm{C}\right)$ \\
\hline Water & 4,18 & 2260 & 100 \\
Guaiacol & 1,98 & 371 & 205 \\
$\mathrm{P}_{666,14}\left[\mathrm{~N}(\mathrm{CN})_{2}\right]$ & 2,18 & & $>300$ \\
\hline
\end{tabular}

